\title{
Pathophysiology and Treatment Options in Trigeminal Meningoceles
}

\author{
Matthias Preuss ${ }^{1} \quad$ Alexander Steinhoff $^{2}$ Constantin J. Zühlke ${ }^{3}$ Dirk Schulz ${ }^{1}$ Marco Stein ${ }^{1}$ \\ Ulf Nestler ${ }^{1}$ Petros Christophis ${ }^{1}$
}

${ }^{1}$ Department of Neurosurgery, Justus-Liebig-University, Giessen, Germany

${ }^{2}$ Department of Neurosurgery, University Leipzig, Leipzig, Germany

${ }^{3}$ Department of Neuroradiology, Justus-Liebig-University, Giessen, Germany

\begin{abstract}
Address for correspondence Matthias Preuss, MD, Department of Neurosurgery, Pediatric Neurosurgery, University Hospital Leipzig, Liebigstrasse 20, D-04103 Leipzig, Germany

(e-mail: preuss@kinderneurochirurgie-leipzig.de).
\end{abstract}

J Neurol Surg Rep 2013;74:81-87.

\begin{abstract}
Keywords

- cerebrospinal fluid (CSF)

- trigeminal meningocele

- spontaneous rhinoliquorrhea

- Sternberg-Cruveilhier canal

- lateral craniopharyngeal canal

Trigeminal meningoceles, lateral to the maxillary nerve (V2), have seldom been reported as underlying pathology for spontaneous rhinoliquorrhea. In contrast to sphenoid meningoceles arising from a persistent lateral craniopharyngeal canal (Sternberg-Cruveilhier, medial to V2), their occurrence seems to be generated by addition of erosive processes to the constitutively thin bony shell underneath the semilunar ganglion, lateral to the round foramen (and V2).

The developmental and anatomical relationships of trigeminal meningoceles to the sphenoid bone are depicted, and in a review of the literature we present the different surgical approaches employed for sealing the dura leak. In view of these techniques we discuss an unusual case of therapy-resistant rhinoliquorrhea with left-sided trigeminal meningocele involving the Meckel cave at the lateral sphenoid and reaching the superior orbital fissure and the medial orbital space.

In contrast to patients who have lateral sphenoidal meningoceles with a persistent lateral craniopharyngeal canal (Sternberg-Cruveilhier), who can be treated successfully using an endoscopic transsphenoidal approach (recurrence rate 13.7\%), the recurrence rate of cerebrospinal fluid (CSF) efflux for trigeminal meningoceles lies much higher (endoscopically $66 \%$, open craniotomy $33 \%$ ). The surgical strategy thus has to be chosen individually, taking into account specific anatomical situations and eventually preceding operations.
\end{abstract}

\section{Introduction}

Among the different entities causing rhinoliquorrhea in closest proximity to the sphenoid bone, trigeminal meningoceles (TMs), lateral sphenoidal meningoceles (LSMs), and the persistence of the lateral craniopharyngeal canal (SternbergCruveilhier canal, SC) have been described.

TMs have only been reported several times in the literature, and nomenclature is still heterogeneous. In general, reports cover various mass lesions in the Meckel cave, ranging from lipoma and meningioma to schwannoma. Only a few deal with meningoceles in the pterygopalatine region, such as in patients with typical stigmata of neurofibromatosis Type 1 (NF 1$)^{1,2}$

TMs are localized in the lateral sphenoid wing lateral to V2 and the foramen rotundum underneath the semilunar ganglion, reaching to the superior orbital fissure, pterygopalatine fossa, and medial orbital space, mostly emerging from an received

December 14, 2012 accepted after revision April 13, 2013

published online

July 12, 2013
Dol http://dx.doi.org/ 10.1055/s-0033-1348955. ISSN 2193-6358. (c) 2013 Georg Thieme Verlag KG
Stuttgart . New York

License terms

(이요 $\Theta \circledast$ 
enlarged Meckel cave and giving rise to osseous erosion of the sphenoid wing.

As a different and more frequent reason for rhinoliquorrhea, LSMs have been described. They lie medially to V2 near the base of the sphenoidal bone $\mathrm{e}^{3-8}$ and can be distinguished from TMs by the absence of orbital or pterygopalatine fossa involvement.

The third dura leak-associated pathology of this region is the developmental anomaly of a persistent SC embryonal canal (lateral craniopharyngeal canal). Still controversially discussed in literature, it has been found in some cases as an underlying condition for LSMs. ${ }^{9-11}$ The canal lies medially to the foramen rotundum and extends from the maxillary nerve $\operatorname{root}(\mathrm{V} 2)$ to a recess of the lateral sphenoid wall, opening into the sphenoid sinus.

\section{Anatomical Considerations}

From an anatomical point of view, there are several "weak spots" in the embryonic development of the sphenoidal bone, which can explain the occurrence of meningoceles or spontaneous rhinoliquorrhea, especially in the area of the trigeminal ganglion and the carotid artery.

The cartilage precursor of the skull base has to form around the cranial nerves, arteries, and veins while respecting their lumen. This can lead to thinned cranial base structures that might be eroded during a lifetime by inflammatory processes or increased intracranial pressure. ${ }^{12}$

The bone formation itself is inhomogeneous. The collision zone between endochondral ossification (lesser wing) and intramembranous ossification (greater wing) lies laterally to the foramen rotundum, extending to the region of the foramen ovale (-Fig. 1). In a computed tomographic (CT) study, 25 patients with lateral sphenoidal cerebrospinal fluid (CSF) leaks were all shown to have the bony defects in this area, lateral to the foramen rotundum. ${ }^{8}$

The sutures between the ossification centers can leave small basal clefts, such as the lateral craniopharyngeal canal

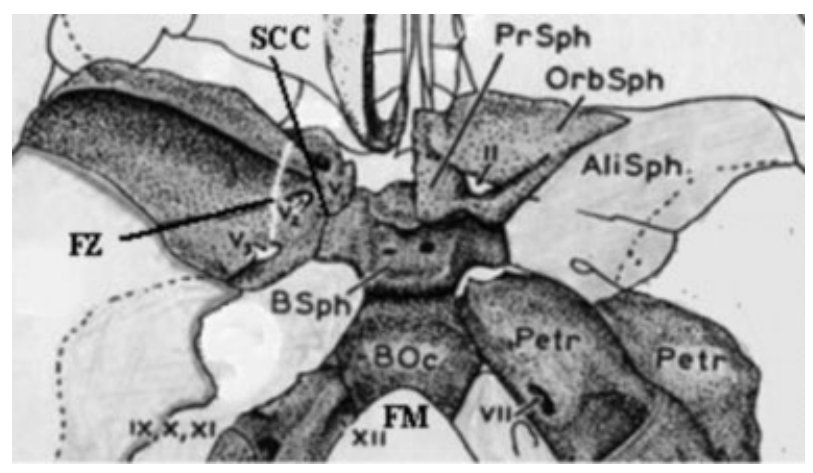

Fig. 1 Cranial base in a 6-month-old embryo shown from above (modified from Fenart and Landouzy) depicting the four main parts forming the sphenoid bone. Cranial nerves: II, V $1, V_{2}, V_{3}$, VII, IX, X, XI, XII. AliSph, alisphenoid; BOc, basioccipitale; BSph, basisphenoid; FM, foramen magnum; FZ, fusion zone between (lateral) and endochondral (medial) ossification; OrbSph, orbitosphenoid; Petr, petrous bone; PrSph, presphenoid; SCC, site of the Sternberg-Cruveilhier canal.
(SC). The canal represents a remnant of the fusion zone between the alisphenoid (greater wing) and the basisphenoid (-Fig. 1). ${ }^{13}$

Development of the sphenoid bone is crucial for the whole cranial base. ${ }^{14-16}$ Distorted midline structure development can result in encephaloceles and palate cleft malformations, ${ }^{17-19}$ and midline basal encephaloceles are known as very rare malformations. ${ }^{11,20}$ Lateral sphenoid malformations give rise to lateral sphenoid meningoceles, as well as to meningoceles of the trigeminal nerve. The meningoceles seem to be associated with sphenoid bone malformations, increased intracranial CSF pressure, and accompanying erosive processes. As shown in the following case description, TMs can result in rhinoliquorrhea and CSF leakage to the pterygopalatine fossa and periorbital fatty tissue, followed by exophthalmos and conjunctivitis together with conjunctival edema.

\section{Nomenclature}

In literature, many different terms have been employed to describe the pathology of a TM. Arachnoid cyst of Meckel's cavity appears to be imprecise, because LSMs also arise from it. The terms transalar sphenoid meningocele and transsphenoidal and transethmoidal meningoceles have been used synonymously in literature. ${ }^{19,20}$

We suggest the term lateral sphenoidal meningocele to describe pathologies medial to V2 and the term trigeminal meningoceles as anatomically more precise for lesions lateral to the foramen rotundum and V2.

\section{Illustrative Case}

A 29-year-old woman presented to the outpatient clinic for rhinoliquorrhea. She also reported a left-sided orbital swelling, especially when bending forward or in prone position. The patient experienced headaches, intermittent rhinoliquorrhea, vomiting, and chronic conjunctivitis. Her first meningitis had been at the age of 17 years, a second occurred a few years later, and both had been treated successfully with antibiotics. A former traumatic head injury was denied and basal cranial fractures had been ruled out with multiple imaging techniques.

Extended neuroradiological imaging included cisternographic magnetic resonance (MR) scan after intrathecal gadolinium-application in prone position. It revealed a left-sided CSF leak along a CSF-containing enlargement of the temporal fossa that extended into the pterygopalatine fossa. CSF was seen in the periorbital fatty tissue and the temporal muscle ( - Fig. 2a). Origin of the fistula was suspected in the temporopolar parasellar region in close proximity to the cavernous sinus and to the Meckel cavity. The clivus, sellar area, and ethmoidal cells appeared to be anatomically altered in the CTscan (-Fig. 2b-d).

The surgical strategy was discussed in the multidisciplinary skull-base board and under suspicion of a temporomedial meningocele of the maxillary nerve, a pterional craniotomy with transsylvian approach and closure of the parasellar 


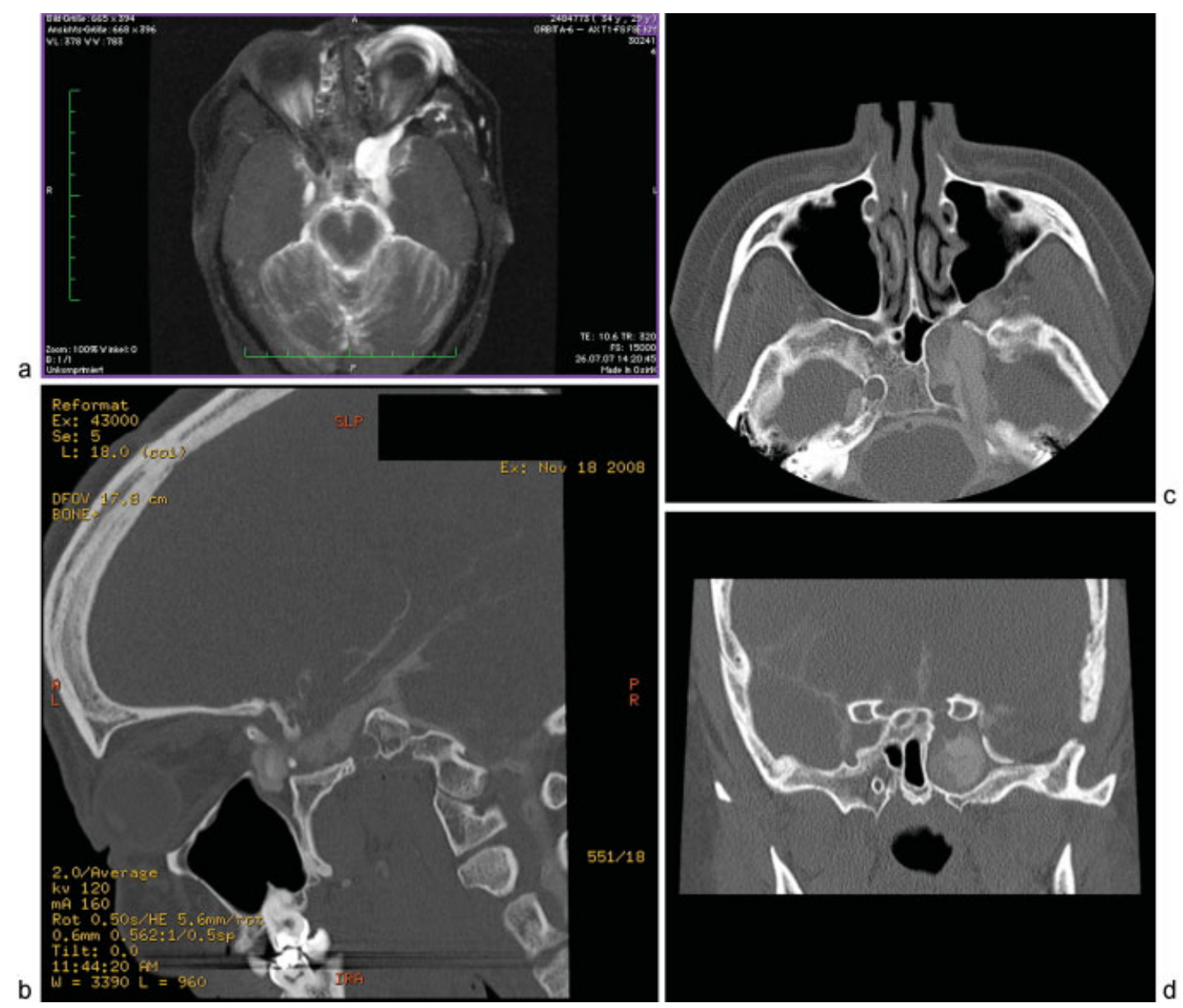

Fig. 2 (a) Preoperative magnetic resonance (MR) T1-weighted axial images enhanced by cisternography. Computed tomography enhanced by cisternography. (b) Oblique reconstruction parallel to the optic canal, (c) axial and (d) coronal reconstruction.

entry point of the meningocele was advised. In a first operation, the suspected entry point of the fistula was closed using multiple layers of subgaleal tissue. The efflux of CSF was stopped but reappeared after several months. The patient then decided to be treated in another neurosurgical department, where a second pterional operation was performed without relieving the symptoms.

About 2 years after first surgery, the patient decided to restart treatment in our institution. Intrathecal contrastenhanced CT revealed the refilled fistula and an enlarged, CSF-containing space in the paraclival region, close to the maxillar nerve and the Meckel cave. A third pterional exploration was proposed, but the patient opted for conservative therapy. Only after increasing orbital swelling and reappearance of rhinoliquorrhea did the patient agree to another intervention.

After this intervention, an oculomotor palsy prompted surgical revision. Fusion of the CT dataset with neuronavigation pictures allowed identification of the entrance of the Meckel cavity. This was covered by dura mater and could be punctured for aspiration of CSF. The wall of the cavity showed a cisternlike arachnoid covering in which the nerve fibers crossing the cavity were partly adhering to the basal arachnoid layer of the cyst and were spread apart. Two walls of the cavity were found, corresponding to arachnoid cystic structures and dural tissue, thus displaying typical criteria for meningoceles. To close the fistula, abdominal fat and muscle tissue were harvested and the periarachnoidal space of the Meckel cave was filled in proximal and distal direction. Hereafter, fat tissue was positioned in the remaining arachnoid space rostrally. Between fat and muscle tissue, liquid dura glue (DuraSeal Xact, Covidien, Mansfield, Massachusetts, USA) was injected into the cavity along the maxillary nerve. Additional muscle was placed on top and covered with fibrin glue. The opening of the cavity was sealed with TachoSil (Takeda Pharmaceuticals; Zurich, Switzerland) and Surgicel (Ethicon, Somerville, New Jersey, USA).

After this intervention, rhinoliquorrhea and orbital swelling disappeared and the third nerve palsy nearly completely recovered within 3 months.

However, about 4 months postoperatively the patient experienced an intermittent CSF leak. Shortly thereafter, orbital swelling and conjunctivitis reoccurred. T2- and CISS 3D T2-scans revealed a partly occluded, yet CSF-containing, meningocele. The patient refused any further interventions, especially implantation of a ventriculoperitoneal (VP) shunt to reduce the intracranial CSF pressure, and she was lost to follow-up. 


\begin{tabular}{|c|c|c|c|c|c|c|c|c|c|c|c|c|c|}
\hline$\geq$ & 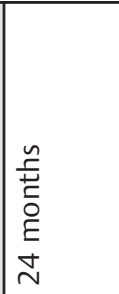 & வ் & 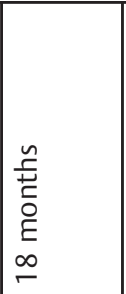 & 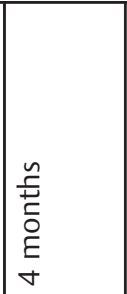 & 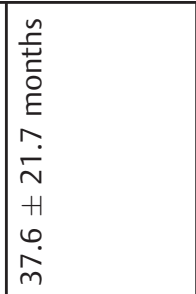 & 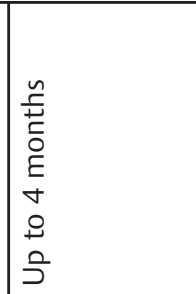 & 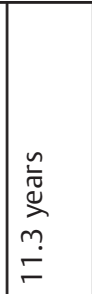 & 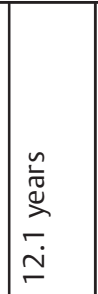 & 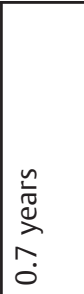 & 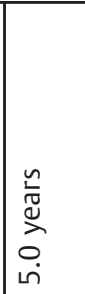 & 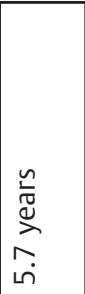 & 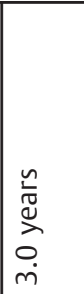 & 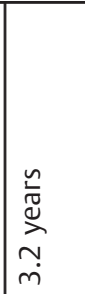 \\
\hline 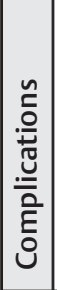 & 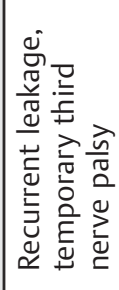 & $\begin{array}{l}\text { İ } \\
\text { ¿े }\end{array}$ & 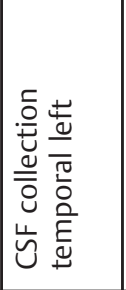 & 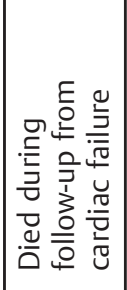 & 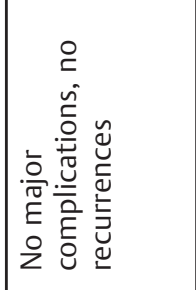 & 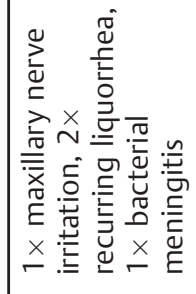 & $\begin{array}{l}0 \\
\check{0} \\
z\end{array}$ & 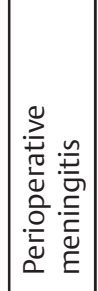 & $\begin{array}{l}0 \\
\tilde{\nu} \\
z\end{array}$ & $\mid \begin{array}{l}0 \\
\check{0} \\
z\end{array}$ & 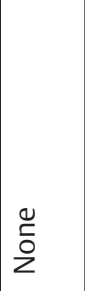 & 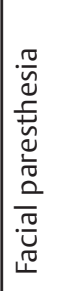 & $\begin{array}{l}0 \\
\check{0} \\
z\end{array}$ \\
\hline 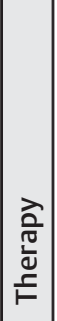 & 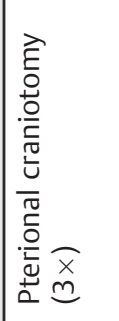 & 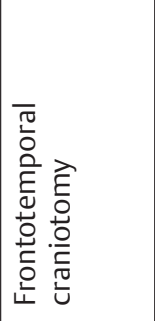 & 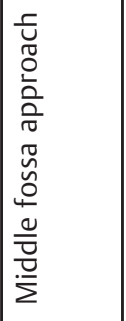 & 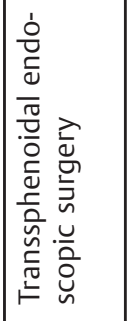 & 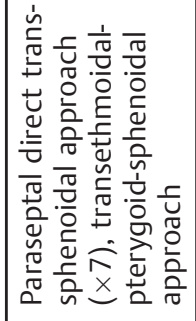 & 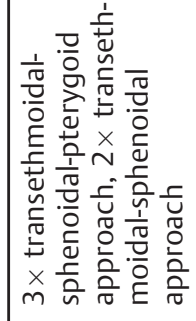 & 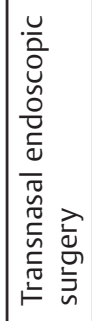 & 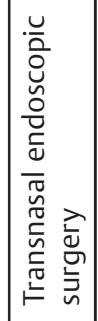 & 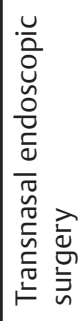 & 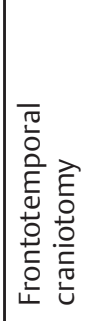 & 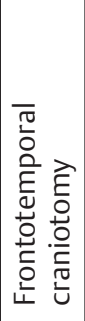 & 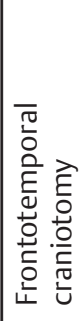 & 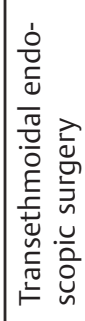 \\
\hline 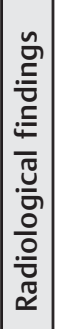 & 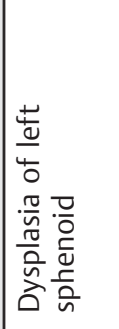 & 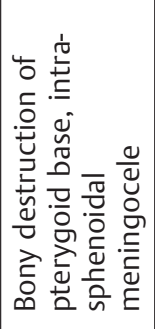 & 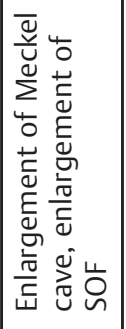 & 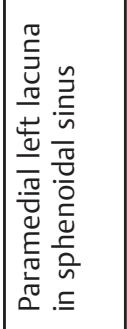 & 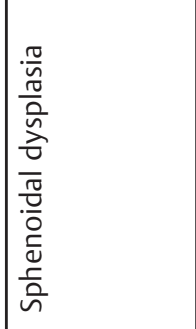 & 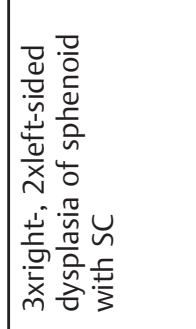 & 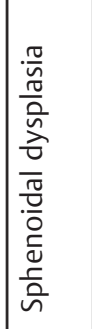 & 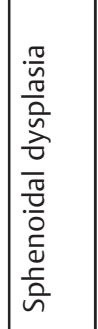 & 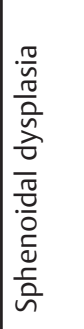 & 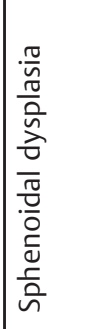 & 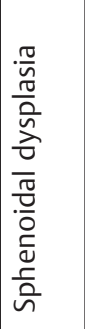 & 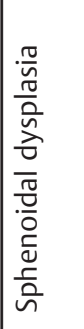 & 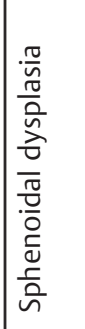 \\
\hline $\mid$\begin{tabular}{|c|}
$\frac{n}{\tilde{y}}$ \\
$\vdots$ \\
$\overline{5}$ \\
$\frac{\pi}{0}$
\end{tabular} & $\begin{array}{l}u \\
\vdots \\
\sum\end{array}$ & $\begin{array}{l}\cup \\
\backsim \\
\sum\end{array}$ & $\sum$ & $\sum$ & $\cup$ & $u$ & $\begin{array}{l}u \\
u \\
\sum \\
u\end{array}$ & $\begin{array}{l}u \\
\tilde{n} \\
\sum \\
\tilde{u}\end{array}$ & $\begin{array}{l}u \\
u \\
\sum \\
u\end{array}$ & $\begin{array}{l}u \\
\tilde{n} \\
\dot{u} \\
\dot{u}\end{array}$ & $\begin{array}{l}u \\
\tilde{u} \\
\sum \\
u\end{array}$ & $\begin{array}{l}u \\
\tilde{n} \\
\vdots \\
u\end{array}$ & $\begin{array}{l}u \\
u \\
\sum \\
u\end{array}$ \\
\hline 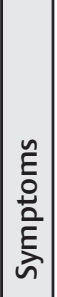 & 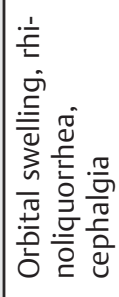 & 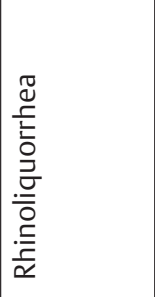 & 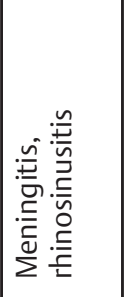 & 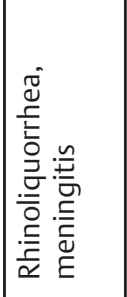 & 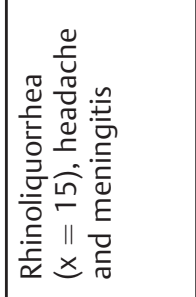 & 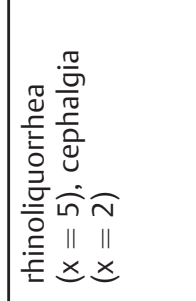 & 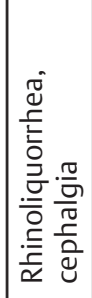 & 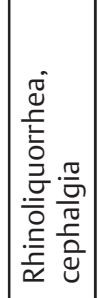 & 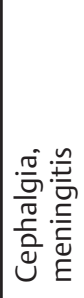 & 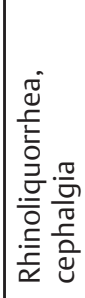 & 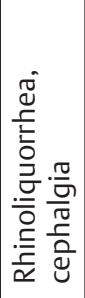 & 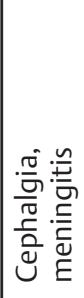 & 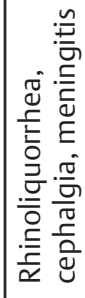 \\
\hline 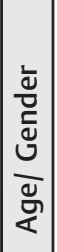 & $\frac{4}{0}$ & $\frac{4}{2}$ & $\frac{\varepsilon}{\infty}$ & $\frac{\omega}{m}$ & 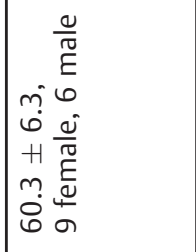 & 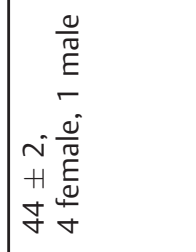 & $\frac{4}{6}$ & $\frac{\omega}{\stackrel{m}{m}}$ & $\frac{4}{\infty}$ & $\underset{f}{\stackrel{4}{f}}$ & $\begin{array}{l}\frac{4}{0} \\
\text { m }\end{array}$ & $\frac{4}{8}$ & $\frac{\omega}{m}$ \\
\hline 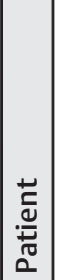 & 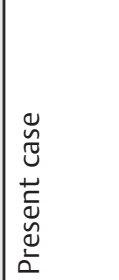 & 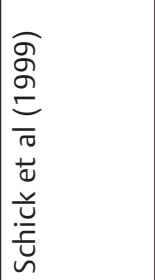 & 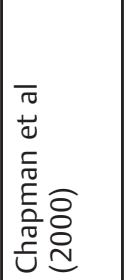 & 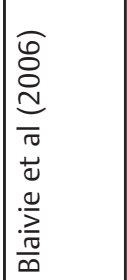 & 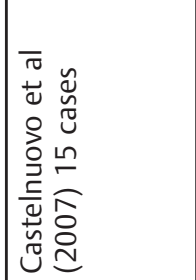 & 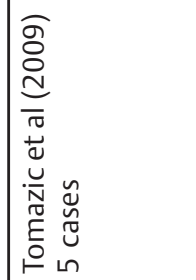 & 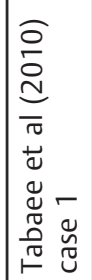 & 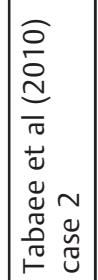 & 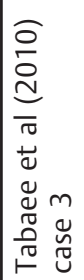 & 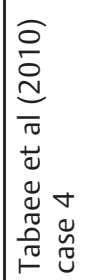 & 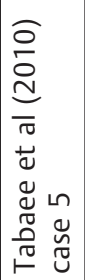 & 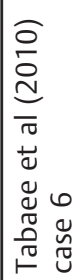 & 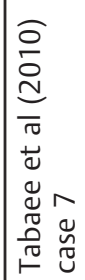 \\
\hline
\end{tabular}




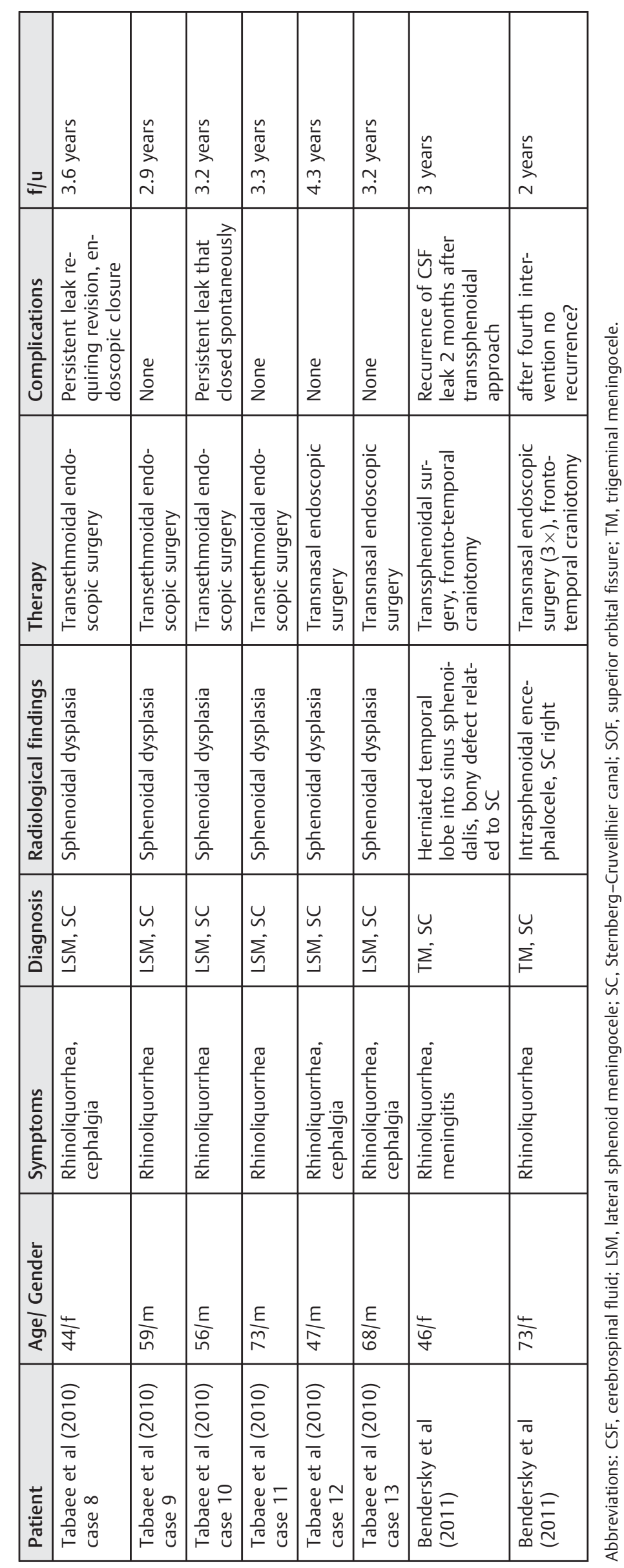




\section{Review of the Literature}

Review of the literature was performed in the Medline database using the search terms trigeminal, encephalocele, Meckel's cave, sphenoid, pterygopalatine, CSF fistula, and Sternberg in all combinations of two keywords.

Search resulted in 38 relevant cases from 23 reports, ranging from 1888 to 2011 (-Table 1). Within these 38 reports, female predominance was found (gender ratio 1.92:1 f:m). Mean age was 52.9 years (age range 8 to 78 years). Patients presented with rhinoliquorrhea in $92.1 \%$ (35 patients), headaches in $34.2 \%$ (13 patients), and meningitis in $15.7 \%$ (6 patients).

Out of 38 patients, 6 had a TM, whereas a persistent SC or LSM was found in 32 patients.

One patient did not receive surgery, leading to persistence of symptoms. A total of 29 patients underwent endoscopic surgery for LSMs, whereas in 3 cases the transcranial approach was preferred. In two cases ${ }^{4}$ transcranial reoperation was performed after endoscopic surgery due to recurrence of CSF leaks. ${ }^{4}$ Recurrence rate was $13.7 \%$ (4 of 29 cases).

From the six TMs, three were treated by an endoscopic transsphenoidal approach, with recurrence of CSF leaks in two of them (66\%). A third case died perioperatively due to cardiac failure during follow-up. One of three patients undergoing craniotomy and open repair experienced recurrent therapy-resistant CSF leakage (present case, 33\%).

Apart from persistent rhinoliquorrhea, the most common complications in the endoscopic group were meningitis (6.3\%) and maxillary nerve irritation (3.1\%). In the craniotomy group, facial paresthesia (3.1\%) and transient diplopia (3.1\%) were encountered.

\section{Discussion}

The case presented here illustrates a rare TM extending from an enlarged Meckel cave into the medial cranial and pterygopalatine fossa, causing therapy-resistant CSF leakage and orbital affection with an unusual collection of CSF in the temporal muscle (-Fig. 2). Although the predominant symptoms of CSF leaks such as rhinoliquorrhea, headache, and meningitis do not help in localizing the dural defect, clinical appearance may vary with the localization of the specific arachnoidal cyst, meningocele, or encephalocele. For example, arachnoid cysts of the region of the Meckel cavity often become symptomatic with facial numbness or pain due to their relation to the trigeminal nerve. ${ }^{21}$

The therapeutic difficulties of that unusual entity have not been solved. In contrast to the far lateral TMs, LSMs and persistent SC can be treated successfully with a transsphenoidal approach. This may be caused by their more medial localization, medially to the foramen rotundum and V2 at the sphenoid base.

Patients with TMs are likely to have a greater benefit from a transcranial approach, which provides better access to the lateral aspects of the Meckel cave and the semilunar ganglion. This is underlined by the higher recurrence rate when using the transsphenoidal approach (-Table 1). Alternatively, transmaxillar transpterygoid approaches may be employed for TMs with a limited pterygoid fossa leakage. ${ }^{1}$

CSF leaks associated with TMs can be assumed to be of idiopathic origin with an elevated intracranial pressure (ICP) as an additional factor. Thus, ICP recording and ICP normalization should be taken into account, and routine use of lumbar drainage as a diagnostic and therapeutic measure has been proposed in lateral sphenoid CSF leaks. ${ }^{22}$

In light of the reviewed literature, together with the presented therapy-resistant case, alternative strategies such as implantation of a VP shunt or temporary lumbar drainage to reduce CSF pressure have to be discussed. The occurrence of the spontaneous CSF leak as part of an idiopathic hydrocephalus syndrome should be considered, with acetazolamide or furosemide being a treatment option. ${ }^{22}$ However, the one case treated conservatively experienced persistence of symptoms, similar to our patient, who experienced recurrence after deciding for nonsurgical follow-up ( $\mathbf{- T a b l e ~} \mathbf{1}$ ). Midfacial degloving has been suggested as approach to the pterygopalatine fossa meningoceles and remains an option for recurrence. ${ }^{21}$

A staged treatment algorithm using lumbar CSF drainage, ICP recording, and invasive location of the skull base defect with intrathecal administration of contrast medium seems to be most promising. Depending on the location and anatomical shape of the TM, open craniotomy or endoscopic transpterygoid approach can be selected. ${ }^{6-8,22}$ Whereas for medial pathologies (SC, LSM) endoscopic transsphenoidal approaches appear to be advantageous, TMs should be primarily considered for an open craniotomy. Alternatively to transcranial approaches, transfacial or transmaxillary/transpterygoid approaches have to be considered as treatment for recurrent, extended TMs involving the pterygopalatine fossa, in which transsphenoidal endoscopic techniques have limited success rates.

In case of therapy-refractory CSF leaks and marked elevation of ICP, VP shunt placement and/or medical treatment should be considered before reintervention. ${ }^{22}$

\section{Conclusion}

Compared with the spontaneous rhinoliquorrhea caused by a persistent SC or LSMs, the point of leakage in TMs lies laterally to V2 and cannot always clearly be visualized, even by sophisticated neuroradiological techniques. This can result in considerable technical difficulties and therefore therapeutic decisions should follow the advice of a multidisciplinary skull base team after taking into account the individual anatomic situation of a patient, preceding operations, and specific risk factors for elevated ICP.

\section{References}

1 Chapman PH, Curtin HD, Cunningham MJ. An unusual pterygopalatine meningocele associated with neurofibromatosis type 1. Case report. J Neurosurg 2000;93(3):480-483

2 de Vries J, Freihofer HPM, Menovsky T, Cruysberg JRM. Successful surgical repair of progressive exophthalmos caused by a 
meningocele in a patient with neurofibromatosis Type 1 . Case report. J Neurosurg 1998;89(6):1032-1035

3 Barañano CF, Curé J, Palmer JN, Woodworth BA. Sternberg's canal: fact or fiction? Am J Rhinol Allergy 2009;23(2):167-171

4 Bendersky DC, Landriel FA, Ajler PM, Hem SM, Carrizo AG. Sternberg's canal as a cause of encephalocele within the lateral recess of the sphenoid sinus: A report of two cases. Surg Neurol Int 2011;2:171

5 Blaivie C, Lequeux T, Kampouridis S, Louryan S, Saussez S. Congenital transsphenoidal meningocele: case report and review of the literature. Am J Otolaryngol 2006;27(6):422-424

6 Castelnuovo P, Dallan I, Pistochini A, Battaglia P, Locatelli D, Bignami M. Endonasal endoscopic repair of Sternberg's canal cerebrospinal fluid leaks. Laryngoscope 2007;117(2):345-349

7 Tabaee A, Anand VK, Cappabianca P, Stamm A, Esposito F, Schwartz TH. Endoscopic management of spontaneous meningoencephalocele of the lateral sphenoid sinus. J Neurosurg 2010;112(5): 1070-1077

8 Tomazic PV, Stammberger H. Spontaneous CSF-leaks and meningoencephaloceles in sphenoid sinus by persisting Sternberg's canal. Rhinology 2009;47(4):369-374

9 Sternberg M. Ein bisher nicht beschriebener Kanal im Keilbein des Menschen. Anat Anz 1888;3:784-785

10 Radoievitch S, Jovanovitch S. [Cruveilhier-Sternberg embryonal canal (lateral craniopharyngeal canal) and its vestiges in the adult human]. Rev Laryngol Otol Rhinol (Bord) 1956;77(3-4):223-232

11 Schick B, Brors D, Prescher A. Sternberg's canal-cause of congenital sphenoidal meningocele. Eur Arch Otorhinolaryngol 2000;257 (8):430-432
12 Wang EW, Vandergrift WA III, Schlosser RJ. Spontaneous CSF Leaks. Otolaryngol Clin North Am 2011;44(4):845-856, vii

13 Sperber GH. Craniofacial Embyrology. Boston: Wright PSG; 1981

14 Michejda M. The role of basicranial synchondroses in flexure processes and ontogenetic development of the skull base. Am J Phys Anthropol 1972;37(1):143-150

15 Laitman JT, Heimbuch RC, Crelin ES. Developmental change in a basicranial line and its relationship to the upper respiratory system in living primates. Am J Anat 1978;152(4):467-482

16 Lieberman DE, McBratney BM, Krovitz G. The evolution and development of cranial form in Homo sapiens. Proc Natl Acad Sci U S A 2002;99(3):1134-1139

17 Jeffery N, Spoor F. Brain size and the human cranial base: a prenatal perspective. Am J Phys Anthropol 2002;118(4):324-340

18 Jeffery N, Spoor F. Ossification and midline shape changes of the human fetal cranial base. Am J Phys Anthropol 2004;123(1):78-90

19 Pollock JA, Newton TH, Hoyt WF. Transsphenoidal and transethmoidal encephaloceles. A review of clinical and roentgen features in 8 cases. Radiology 1968;90(3):442-453

20 Soyer P, Dobbelaere P, Reizine D, Ferquel C. Transalar sphenoidal meningoencephalocele associated with buccal angiomatosis. One case. J Neuroradiol 1990;17(3):222-226

21 Kantas I, Tzindros G, Papadopoulou A, Marangos N. Midfacial Degloving: The Best Alternative for Treatment of Trans-sphenoidal Meningocele of the Pterygopalatine Fossa. Skull Base 2006;16 (2):117-122

22 Alexander NS, Chaaban MR, Riley KO, Woodworth BA. Treatment strategies for lateral sphenoid sinus recess cerebrospinal fluid leaks. Arch Otolaryngol Head Neck Surg 2012;138(5):471-478 